\title{
MOPTOP: a multi-colour optimised optical polarimeter
}

\author{
Helen Jermak ${ }^{\mathrm{a}}$, Iain A. Steele ${ }^{\mathrm{a}}$, and Robert J. Smith ${ }^{\mathrm{a}}$ \\ ${ }^{a}$ Astrophysics Research Institute, Liverpool John Moores University, Liverpool Science Park \\ IC2, 146 Brownlow Hill, Liverpool. L3 5RF.
}

\begin{abstract}
We present the design and science case for the Liverpool Telescope's fourth-generation polarimeter; MOPTOP: a Multicolour OPTimised Optical Polarimeter which is optimised for sensitivity and bi-colour observations. We introduce an optimised polarimeter which is as far as possible limited only by the photon counting efficiency of the detectors. Using a combination of CMOS cameras, a continuously rotating half-wave plate and a wire grid polarising beamsplitter, we predict we can accurately measure the polarisation of sources to $\sim 1 \%$ at $\sim 19$ th magnitude in 10 minutes on a 2 metre telescope. For brighter sources we anticipate much low systematics $(\lesssim 0.1 \%)$ than our current polarimeter. The design also gives the ability to measure polarization and photometric variability on timescales as short as a few seconds. Overall the instrument will allow accurate measurements of the intra-nightly variability of the polarisation of sources such as gamma-ray bursts and blazars (AGN orientated with the jet pointing toward the observer), allowing the constraint of magnetic field models revealing more information about the formation, ejection and collimation of jets.
\end{abstract}

Keywords: optical polarimetry, instrumentation, beamsplitter, CMOS cameras

\section{INTRODUCTION}

The accurate measurement of rapidly varying polarization in jetted sources is an important technique in astronomy. To probe the structure and emission regions of active galaxy jets, the exploration of spectral polarimetric and photometric variation during periods of flaring and quiescence are vital. One of the leading simultaneous multicolour polarimeters is the Ringo $3^{1}$ polarimeter on the fully autonomous and robotic 2 metre Liverpool Telescope. ${ }^{2}$ Ringo3 consists of a rapidly rotating Polaroid (1 rotation every $\sim 2.5$ seconds) which modulates the incoming beam of light and using dichroic mirrors, distributes the light in three wavelength bands to three electron-multiplying charged coupled devices (EMCCDs). Ringo3 has been extensively used for polarimetric and photometric studies of the optical section of the spectral energy distribution of beamed active galaxy sources known as blazars. These results, along with others from Ringo3 analysis, contribute to the design requirements of a new, optimised polarimeter (MOPTOP) which we present here. We aim to create a polarimeter which is limited by photon-counting statistics only rather than instrumental systematic effects.

\section{SCIENCE CASE}

The emerging importance of time domain astrophysics was recognized with the 2012 Royal Society meeting New windows on transients across the universe and the subsequent meeting at the Kavli centre on "Interpreting signals from astrophysical transient experiments". Linear polarisation is now taking a leading role as a key diagnostic of physical conditions (for example magnetic field strength and geometry and relativistic plasma dynamics) in such transient sources as blazars, active galactic nuclei, x-ray binaries and gamma ray bursts (GRBs). As an example, use of polarimetry as a diagnostic tool in time domain programmes has increased by a factor 5 on the Liverpool Telescope over the period 2012-2015. In all of these high energy sources, polarisation allows astronomers to probe the physical conditions at spatial scales that will never be accessible to direct imaging observations. For example the Ringo2 polarimeter was used to make the first ever detections of polarisation in $\mathrm{GRBs}^{3}$ and its

Further author information: (Send correspondence to Helen Jermak)

Helen Jermak: E-mail: h.e.jermak@2012.ljmu.ac.uk

Iain A. Steele: E-mail: I.A.Steele@ljmu.ac.uk

Robert J Smith : E-mail : r.j.smith@ljmu.ac.uk 
time evolution. ${ }^{4}$ Ringo2 and Ringo3 have also been used for leading programmes in Blazar variability. ${ }^{5}$ It has also made the first high precision ( \pm 2 degrees) measurements of the inclination angle of the disk in a high mass $\mathrm{X}$-ray binary. ${ }^{6}$

For solar system sources such as asteroids and comets, the most important feature of a polarimeter is than it can measure low degrees of polarisation to an excellent accuracy (i.e is free from systematic errors). For comets, accurately measuring the polarisation strength can map the structure of a comet from the solar light scattered by dust particles in the coma. The linear polarisation depends on the intrinsic properties of the dust particles (their size, structure and composition) and also on their spatial distribution. The wider views of polarised light in the coma provide a study of the dust population distribution not available to the point sample, in-situ measurements found by spacecraft such as the ESA Rosetta mission. Scattering is strongly wavelength dependent, making a multi-colour approach important. Similarly for the flood of new asteroids (many 10s per day) being discovered by the ESA Gaia Mission, polarimetric measurements at different phase angles can give the first measurement of the distribution of geometric albedo and surface roughness of in large sample asteroid populations. ${ }^{7}$

A particular challenge presented by the GRB and blazar sources is the short timescales associated with them. For GRBs the optical afterglow of the fireball fades on timescales of minutes ${ }^{8}$ therefore both a rapid response and an observing approach that gathers maximum information in the time available is essential. This implies the need for maximum sensitivity combined with simultaneous, multicolour capability. By following the evolution of the light curve at several wavelengths as the synchrotron frequency evolves through the optical waveband this allows us to distinguish between forward and reverse travelling (in the co-moving frame) shocks. This is important as the reverse shock explores the magnetic field structure in the jet and the forward shock that in the ambient media into which the jet is expanding.

The contribution of different components of an active galaxy to the overall bolometric flux can vary during periods of quiescence and flaring. The structure, conditions and properties of the components cause them to emit radiation at different wavelengths and consist of thermal and/or non-thermal radiation. When studying the jet of active galactic sources that are orientated on the sky so that their jet axes are within an opening angle of $\sim 10^{\circ}$ of the observer, it is not possible to resolve the jet or emitting region so the exploration of the point source object is through the total flux which is dominated by synchrotron emission from the highly relativistic, Doppler boosted and rapidly varying jet.

Simultaneously measuring multiple sections of the optical energy distribution of blazars over long time periods allows the exploration of the spectral changes in the source which afford information about the energy of the electron emitting synchrotron radiation, the luminosity of the accretion disk and can help study the physical properties of different blazar subclasses (which have been classified by observational properties). An example multicolour blazar lightcurve from Ringo3 is presented in Figure 1 Polarimetric measurements explore the magnetic field within the jet. The relationship between the electric vector position angle rotations (which can be as long as $\sim 700^{\circ}$ in some sources ${ }^{5,9}$ ). Flaring activity in optical wavelength bands can help explore the relationship between emitting regions of the jet and the formation of shocks.

\section{LESSONS LEARNED FROM RINGO3}

Since 2006 the Liverpool Telescope group have been working on a programme of research and development in instrumentation for time domain polarimetry and have developed a series of polarimeters of increasing sensitivity and wavelength coverage and exploring different polarimetric techniques.

The current polarimeter, RINGO3, is a multicolour device fitted with dichroic mirrors that split the polarised incoming beam of light into three optical wavebands: blue-visible $(350-640 \mathrm{~nm})$, green $(650-760 \mathrm{~nm})$ and red $(770-1000 \mathrm{~nm}) .{ }^{1}$ This allows the measurement of polarisation degree and angle in three colours simultaneously. Table 1 shows the wavelength ranges for each of the three cameras.

The combination of a rapid rotating Polaroid and two dichroic mirrors introduced an unforeseen instrumental polarisation which could not be characterised by studying standard sources as the instrumental polarisation varied as a function of field rotation of the telescope. Since dichroic mirrors are successfully used in other polarimeters (e.g. DIPOL- $2^{10}$ ) this non-characterisable error was attributed to an interaction between the strongly polarised and time variable output from the Polaroid (even for an un-polarised source) and the dichroic mirror coatings. 
$0 j 287$

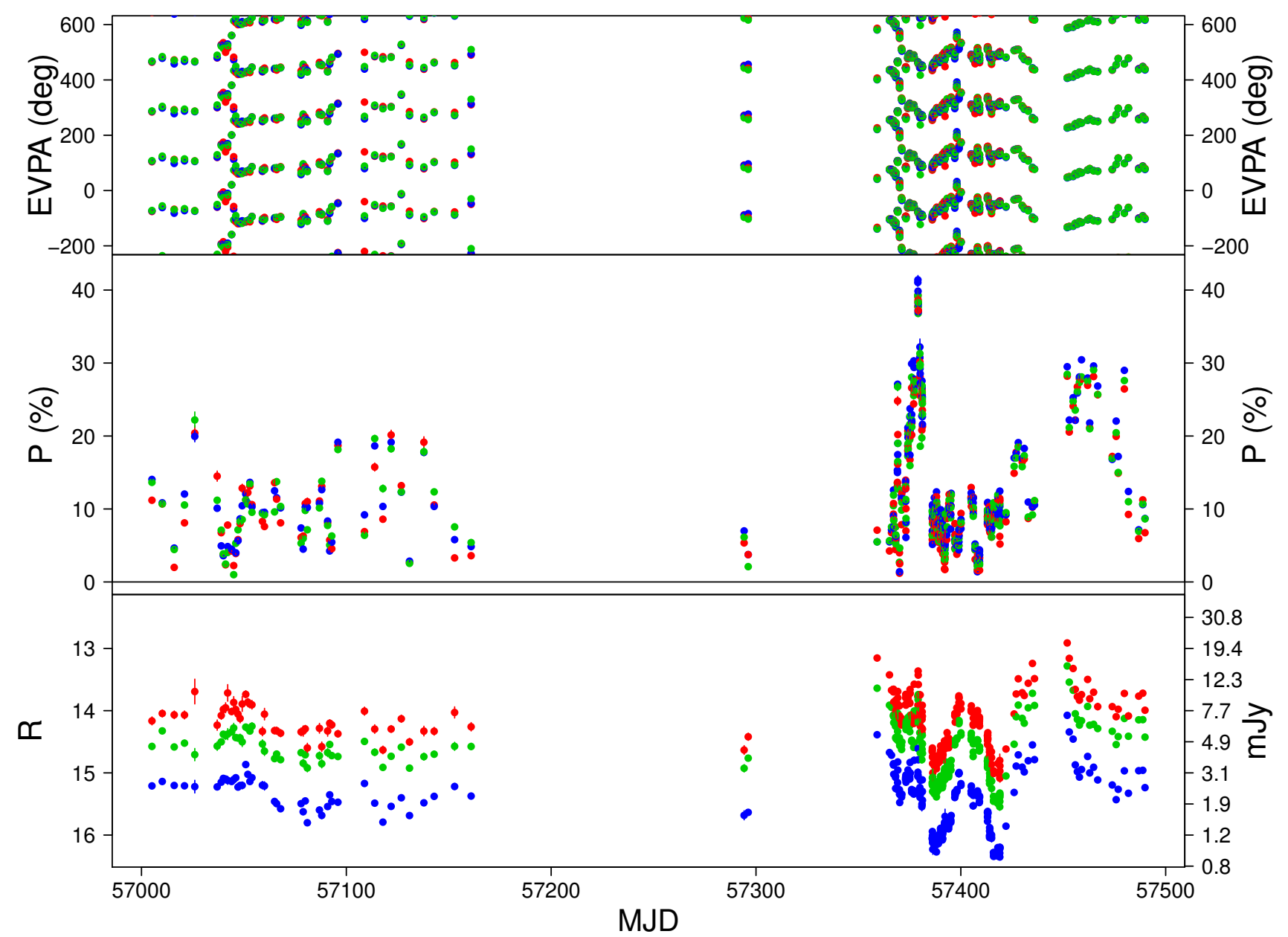

Figure 1. Electric Vector Polarization Angle, Degree of Polarization and $b^{*}, g^{*}$ and $r^{*}$ magnitudes as a function of time for the Blazar OJ287. All data was obtained with the Ringo3 polarimeter on the Liverpool Telescope. By studying the evolution of EVPA, the passage of emitting material through regions of different magnetic field order and orientation can be traced. By studying the evolution of the degree of polarization and magnitude at different wavelengths, the time dependant contributions of jet and disk emission can be followed.

Table 1. Ringo3 specifics for each camera, field of view and wavelength range. The path describes whether the light travels through or is reflected by the dichroic mirrors.

\begin{tabular}{|l|l|l|l|}
\hline RINGO3 & red & green & blue \\
\hline Field of view (diameter) & 5.9 arcmin & 4.1 arcmin & 4.1 arcmin \\
\hline$\lambda$ range & $770-1000 \mathrm{~nm}$ & $650-760 \mathrm{~nm}$ & $350-640 \mathrm{~nm}$ \\
\hline Path & $1 \mathrm{x}$ transmission & 2 reflections & 1 reflection, 1 transmission \\
\hline
\end{tabular}




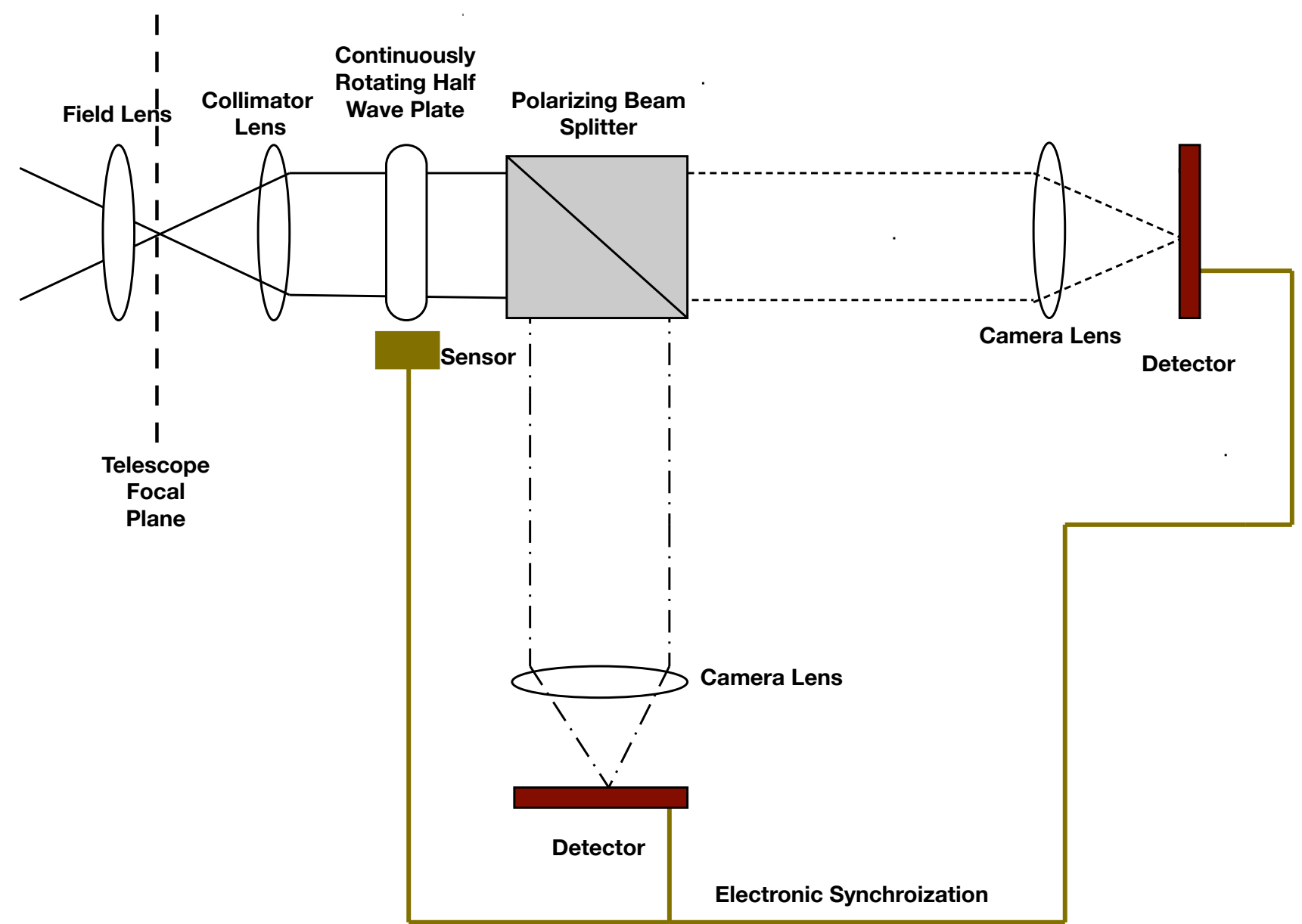

Figure 2. Schematic Layout of 'mini'-MOPTOP illustrating the design principal and showing the principal components. The incoming white light beam from the telescope is indicated with solid, black lines. A continuously rotating half wave plate modulates the polarisation angle of the incoming beam. One polarisation state is transmitted (dashed line) and the other reflected (dotted line) by the beam splitter. Each polarisation state is then simultaneously recorded by fast readout CMOS cameras synchronized to the wave plate angle.

For Ringo 3 to resolve this problem a quasi-depolarising optic was introduced to the collimator lens to disrupt the polarised signal and produce 'scrambled' light. Since the polarisation was measured according to the intensity of light in each rotor position, scrambling the polarisation signal after the collimator lens did not prevent the measurement of polarisation. While providing a degree of correction, there are still small systematic errors remaining due to this effect. This limits the systematic error performance of Ringo3 to $\sim 0.5 \%$.

\section{DESIGN CONCEPT}

\subsection{Optical Design}

Our new design aims to combine the advantages of a traditional dual beam polarimeter in control of systematic errors with the high time resolution of our Ringo series of designs. An outline of the design concept showing the components necessary to make polarization measurements in a single band ('mini'-MOPTOP) is presented in Figure 2.

The optical design of the new polarimeter is such that the incoming beam of light enters the modulator element, a continuously revolving half-wave plate, which rotates the polarization angle of the incoming beam before it hits a wire grid polarising beam splitter which divides the beam into the $\mathrm{p}$ and s polarisation states. The 
two polarization states are them imaged onto two fast readout, low noise imaging cameras which are electronically synchronized to the wave plate angle.

The combination of a half wave plate and beamsplitter instead of using a rotating Polaroid filter allows the exploitation of $\sim 100 \%$ of the incoming beam of light (compared with the $\sim 50 \%$ transmission of the Ringo3 Polaroid). This immediately increases the sensitivity of the instrument.

By rotating a half wave plate by 22.5 degrees, the polarisation angle of incident radiation can be rotated by 45 degrees. Unlike other polarimeter designs however, we will not step the plate between certain fixed positions (which has associated efficiency overheads) but instead rotate the wave plate continuously at $\sim 15 \mathrm{rpm} .16$ points around the edge of the wave plate will trigger a fast proximity sensor every 22.5 degrees of rotation. This trigger will synchronize the exposures to the fast readout cameras. Calculations show that using a continuous (as opposed to stepped) rotation has no effect on the accuracy of the measured polarisation. We have confirmed this with our simpler 8 position continuously rotating Polaroid system in RINGO2 $\left({ }^{11}\right)$. As well as increased observing efficiency, this use of rapid and continuous rotation is essential when observing rapidly variable objects. As an example, a Gamma ray Burst optical counterpart can fade by $20 \%$ in one minute. A conventional, slowly stepped wave plate would misinterpret this variability as a polarisation signal of similar magnitude (since polarimetry is essentially a difference imaging technique). However the high time resolution of MOPTOP ( 1 second) means only a $0.3 \%$ error would be introduced into such measurement even when the data are subsequently temporally binned over the same one minute time period.

The beam-splitter separates the beam into $\mathrm{s}$ and $\mathrm{p}$ polarisation states to be recorded on separate cameras. With the half wave plate in its initial position, the difference between the two images equals the Stokes Q parameter. The difference images at the subsequent half wave plate position (22.5 degrees) equals the Stokes U parameter. The third wave plate position difference yields $-\mathrm{Q}$ and the fourth $-\mathrm{U}$. The sum of all the images equals the Stokes I parameter. Suitable wire grid beam-splitters are available with very high throughput $(>90 \%)$ for maximum sensitivity, a wide range of acceptance angles ( \pm 10 degrees) for minimal field dependant instrumental depolarisation and a very high contrast ratio (>2000:1) over a wavelength range from 380 to $800 \mathrm{~nm}$ for very low overall instrumental depolarisation $(>0.1 \%)$. As already described, by using a beam splitter in this role rather than a Polaroid we achieve double the throughput. We will also have much better control of systematic errors since our individual Stokes parameters will be formed from simultaneously obtained difference images and the rapidly alternating measurements of opposite signs of $\mathrm{Q}$ and $\mathrm{U}$ allows cancellation of offset biases and flat field errors.

\subsection{CMOS Imaging Cameras}

In RINGO2 we pioneered the use of fast readout, low noise detectors in a polarimeter. Such detectors have two key advantages in this application. Firstly they are able to observe rapidly varying sources at greater sensitivity. Secondly, and most importantly, the very low readout noise allows data to be taken a high frame rates $(\sim 0.1-1 \mathrm{~Hz})$. This provides excellent temporal resolution when the source is sufficiently bright or, when the source is faint, allows temporal binning of high frame rate data without a significant read noise penalty to achieve the desired polarimetric accuracy. This gives huge dynamic range $(\sim 100000: 1)$ and the resulting ability to observe transients of unknown and rapidly variable brightness. For RINGO2 and 3 we achieved the required low noise, fast readout using then state of the art Electron Multiplying CCD (EMCCD) technology. However due to their mode of operation EMCCDs suffer charge multiplication noise which causes a factor 2 reduction in the instrument sensitivity. For MOPTOP we will use newly available scientific CMOS (sCMOS) detectors for the first time in astronomical polarimetry. We have already carried out tests that show their readout can be synchronized electronically to the degree of precision necessary. ${ }^{12}$ sCMOS detectors maintain the rapid readout $(>100 \mathrm{fps})$ and ultra low read noise ( $<0.9$ electrons) advantages of EMCCD but add much greater sensitivity ( $>80 \%$ vs $\sim 40 \%$ for EMCCDs). In addition sCMOS detectors are available in much larger formats ( $\sim 4$ Mpix vs $\sim 1$ Mpix from EMCCDs) allowing the full 8 arcmin telescope field of view to be sampled at optimal (Nyquist sampled) resolution. 


\section{MOPTOP}

The previous polarimeters Ringo, Ringo2 and the current Ringo3 have been mounted on the Liverpool Telescope which is the worlds largest fully robotic telescope. This is key in enabling them to responding autonomously to triggers from transient detection experiments such as the NASA Swift Satellite and the LIGO and VIRGO gravitational wave detectors. Response times for Gamma Ray Bursts are 2-3 minutes from satellite detection to our ground based optical observations, depending on the object location on the sky.

The design of MOPTOP takes our capability to a new level by meeting many ideal requirements (see below). It takes a number of already novel aspects from the RINGO series (a fast rotating element to allow high time resolution; the use of fast readout, very low noise cameras allowing a unique, post data acquisition, signal to noise ratio optimization; the use of dichroic mirrors to provide multi-wavelength capability) and adds a unique optical dual-camera configuration to minimize systematic errors and provide the highest possible sensitivity.

We have identified the following requirements for the optimised polarimeter:

- Maximum Sensitivity, such that the polarimetric accuracy obtained is dominated by the photon counting (Poisson) statistics of the light incident on the telescope.

- Very low systematic errors $(<0.1 \%)$ allowing measurement of low polarisation sources.

- Ability to measure a rapidly ( $\sim 10$ seconds) variable or fading source.

- Ability to measure all sources in the field of view without prior identification.

- Ability to measure sources over the widest possible dynamic range of photon counts and without prior knowledge of the source brightness.

- Ability to measure polarisation over a number of different wavelength ranges simultaneously.

- Be available to respond to transient events and other targets of opportunity.

- The widest possible field of view (as limited by the telescope optics).

- The best possible spatial resolution (as. limited by atmospheric effects).

While dual beam polarimeters have been built before, they have always combined the signal onto a single camera. This causes problems with source overlap and reduced sensitivity (since each object effectively receives double the background sky noise contribution compared to normal imaging). The traditional solution to this has been the use of a focal plane mask, but this reduces the field of view and means the instrument can not be used when the object localization on the sky is poor (which is often the case for transient sources). Our dual camera design avoids these problems, providing full sensitivity with the full field of view.

Overall we anticipate the design to have four times the sensitivity of Ringo3 (a factor of two from the switch from Polaroid to beam splitting optics and a factor of two from the switch from EMCCD to sCMOS cameras). This implies a polarization accuracy of $\sim 1 \%$ should be obtainable with a 10 minute exposure at $19^{\text {th }}$ magnitude. An example of how the MOPTOP design can be adapted to accommodate two sets of cameras for two colour operation is shown in Figure 3. An important advantage of the MOPTOP design over our previous Ringo3 polarimeters is that the polarization angle of the incoming light to the dichroic mirrors is fixed (rather than rotating). This should eliminate the major cause of residual systematic error identified in Ringo3. This, plus the intrinsic advantage of a dual beam design in allowing differential correction of atmospheric and other time domain systematic effects, means that for brighter objects, we anticipate the dual beam design should reduce systematic errors by a factor of 4 over Ringo3, reducing them to $\sim 0.1 \%$. 


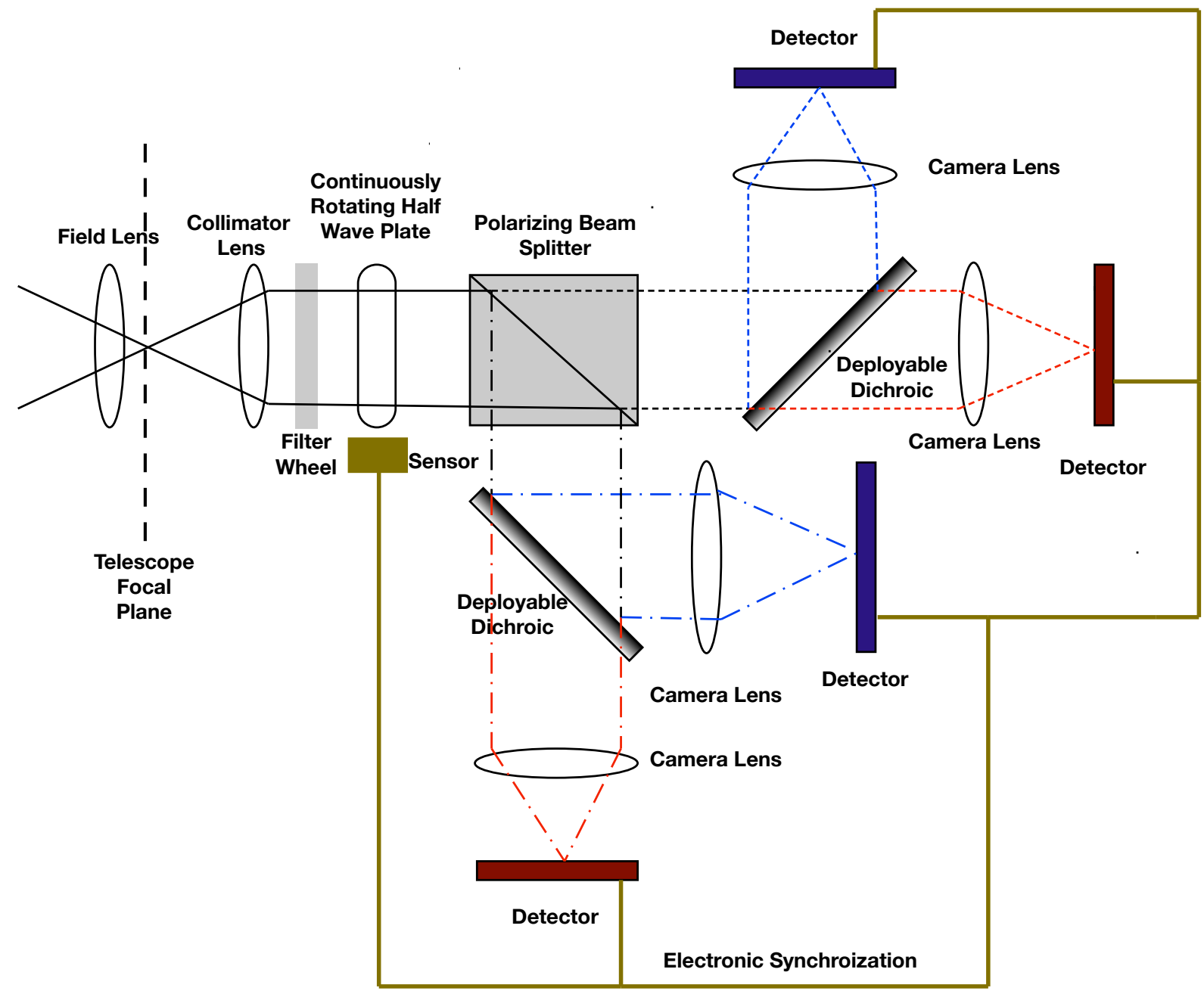

Figure 3. Schematic 2D rendering of a 2 camera set version of MOPTOP showing the principal components. The incoming white light beam from the telescope is indicated with solid, black lines. A continuously rotating half wave plate modulates the polarisation angle of the incoming beam. One polarisation state is transmitted (dashed line) and the other reflected (dotted line) by the beam splitter. Each polarisation state is then split by a dichroic mirror before being simultaneously recorded by fast readout CMOS cameras synchronized to the wave plate angle. Alternatively the dichroic mirrors may be removed from the beam and a specific wavelength range selected using the filter wheel. 

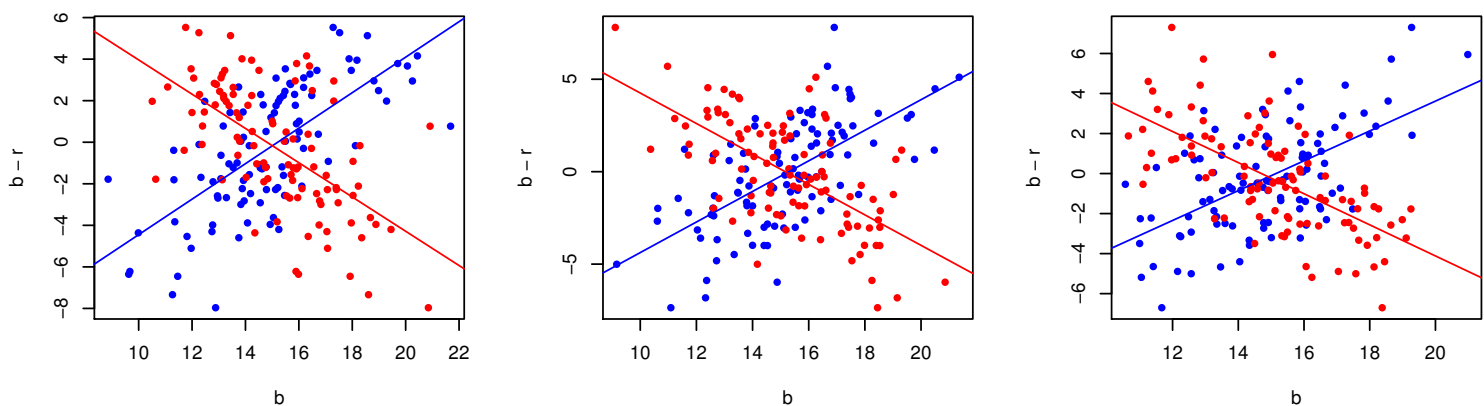

Figure 4. Plots of randomly generated identical $b$ and $r$ values with independent random errors in the form $b$ vs $b-r$ (blue) and $r$ vs $b-r$ (red). This shows three iterations of the code and the false tendency of the fit to be positive (blue) or negative (red) depending on which quantity ( $b$ or $r$ is plotted on the $\mathrm{x}$-axis.

\section{TWO VS THREE BAND DESIGNS}

The standard practice when exploring the variable spectral properties of blazars is to plot (B-V) vs V, (V-R) vs $\mathrm{V},(\mathrm{R}-\mathrm{I})$ vs $\mathrm{V}$ and $(\mathrm{B}-\mathrm{I})$ vs $\mathrm{V}$ using the Johnson and Cousin filters (e.g. $\left.{ }^{13}\right)$. Or, depending on the wavelength (J-I) vs J , (J-R) vs R (e.g. $\left.{ }^{14}\right)$.

However, it is not widely appreciated that a 2-band approach for the study of spectral properties vs magnitude is susceptible to producing false correlations. As an example, following the same practice with our Blazar sample using the (non-standard) Ringo3 optical wavebands: blue-visible 'b*' (350-640 nm), green ' $\mathrm{g} *$ ' (650-760 nm) and red ' $\mathrm{r}^{*}$ ' $(770-1000 \mathrm{~nm})\left({ }^{1}\right)$ we found that the blazars seemed to show either a 'redder' or 'bluer' when brighter property depending on the magnitude or degree of polarisation used on the $\mathrm{x}$ axis.

This was explored further with a rudimentary code which generates two variables $(b$ and $r)$ of identical random magnitude but different errors values and plots these variables against each other in the form $b, b-r$ and $r, b-r$ (similar to the form taken in the colour-magnitude plots). A linear regression fitted to each set of data-points and shows the preference for false fits (dependent on the $\mathrm{x}$ axis colour). Figure 4 shows an example of 3 runs with 100 magnitude values centred on $b=r=15.0$ with a Gaussian $\sigma=1$ and 100 error values with $\sigma=2$. Depending on which value is plotted on the $x$ axis, either a bluer when brighter or redder when brighter trend is seen.

This highlights the necessity to have an independent magnitude (i.e. one not contained in the colour index) on the $\mathrm{x}$ axis to avoid false trends in the data caused by outliers. For this reason MOPTOP's design will be extended to include at least 3 cameras.

\section{CONCLUSION}

We have presented how a unique combination of CMOS cameras, a continuously rotating half-wave plate and a wire grid polarising beamsplitter can be used to create a multicolour, optimised polarimeter which utilises $\sim 100 \%$ of the incoming light while retaining high time resolution. We have begun testing the synchronization of sCMOS cameras ${ }^{12}$ necessary for the design. Our next step will be to do laboratory testing of the single band 'mini'-MOPTOP design before going on to build a multi-band version. To eliminate systematic errors in colour-brightness investigations we envisage the final MOPTOP design will have at least 3 wavebands.

\section{REFERENCES}

[1] Arnold, D. M., Steele, I. A., Bates, S. D., Mottram, C. J., and Smith, R. J., "RINGO3: a multi-colour fast response polarimeter," in [Society of Photo-Optical Instrumentation Engineers (SPIE) Conference Series], Society of Photo-Optical Instrumentation Engineers (SPIE) Conference Series 8446 (Sept. 2012).

[2] Steele, I. A., Smith, R. J., Rees, P. C., Baker, I. P., Bates, S. D., Bode, M. F., Bowman, M. K., Carter, D., Etherton, J., Ford, M. J., Fraser, S. N., Gomboc, A., Lett, R. D. J., Mansfield, A. G., Marchant, J. M., Medrano-Cerda, G. A., Mottram, C. J., Raback, D., Scott, A. B., Tomlinson, M. D., and Zamanov, R., "The Liverpool Telescope: performance and first results," in [Ground-based Telescopes], Oschmann, Jr., 
J. M., ed., Society of Photo-Optical Instrumentation Engineers (SPIE) Conference Series 5489, 679-692 (Oct. 2004).

[3] Steele, I. A., Mundell, C. G., Smith, R. J., Kobayashi, S., and Guidorzi, C., "Ten per cent polarized optical emission from GRB090102," Nature 462, 767-769 (Dec. 2009).

[4] Mundell, C. G., Kopač, D., Arnold, D. M., Steele, I. A., Gomboc, A., Kobayashi, S., Harrison, R. M., Smith, R. J., Guidorzi, C., Virgili, F. J., Melandri, A., and Japelj, J., "Highly polarized light from stable ordered magnetic fields in GRB120308A," Nature 504, 119-121 (Dec. 2013).

[5] Jermak H. et al. MNRAS, in press (2016).

[6] Słowikowska, A. et al MNRAS, submitted (2016).

[7] Belskaya I. et al. Proc. ESO Gaia-Fun SSO Workshop, 103+ (2014).

[8] Piran, T., "Gamma-ray bursts and the fireball model," Physics Reports 314, 575-667 (June 1999).

[9] Marscher, A. P., Jorstad, S. G., Larionov, V. M., Aller, M. F., Aller, H. D., Lähteenmäki, A., Agudo, I., Smith, P. S., Gurwell, M., Hagen-Thorn, V. A., Konstantinova, T. S., Larionova, E. G., Larionova, L. V., Melnichuk, D. A., Blinov, D. A., Kopatskaya, E. N., Troitsky, I. S., Tornikoski, M., Hovatta, T., Schmidt, G. D., D'Arcangelo, F. D., Bhattarai, D., Taylor, B., Olmstead, A. R., Manne-Nicholas, E., Roca-Sogorb, M., Gómez, J. L., McHardy, I. M., Kurtanidze, O., Nikolashvili, M. G., Kimeridze, G. N., and Sigua, L. A., "Probing the Inner Jet of the Quasar PKS 1510-089 with Multi-Waveband Monitoring During Strong Gamma-Ray Activity," Astrophysical Journal Letters 710, L126-L131 (Feb. 2010).

[10] Piirola, V., Berdyugin, A., and Berdyugina, S., "DIPOL-2: a double image high precision polarimeter," in [Ground-based and Airborne Instrumentation for Astronomy V], Proc. SPIE 9147, 91478I (Aug. 2014).

[11] Steele, I. A., Bates, S. D., Guidorzi, C., Mottram, C. J., Mundell, C. G., and Smith, R. J., "RINGO2: an EMCCD-based polarimeter for GRB followup," in [Society of Photo-Optical Instrumentation Engineers (SPIE) Conference Series], Society of Photo-Optical Instrumentation Engineers (SPIE) Conference Series 7735 (July 2010).

[12] Steele I.A. et al., "Experiments with synchronized sCMOS cameras," Proc SPIE - this conference (2016).

[13] Gaur, H., Gupta, A. C., Strigachev, A., Bachev, R., Semkov, E., Wiita, P. J., Peneva, S., Boeva, S., Slavcheva-Mihova, L., Mihov, B., Latev, G., and Pandey, U. S., "Optical flux and spectral variability of blazars," MNRAS 425, 3002-3023 (Oct. 2012).

[14] Zhang, B.-K., Zhou, X.-S., Zhao, X.-Y., and Dai, B.-Z., "Long-term optical-infrared color variability of blazars," Research in Astronomy and Astrophysics 15, 1784 (Nov. 2015). 